\title{
Le passé, le présent et un avenir très radieux
}

par Glen J Pearson

\begin{abstract}
Hier n'est plus. Demain n'est pas encore. Nous n'avons qu'aujourd'hui. Mettons-nous à l'aeuvre.
\end{abstract} -Mère Teresa

$\mathrm{L}$ 'histoire de la pharmacie au Canada a 400 ans. Elle débute le 15 juillet 1617 avec l'arrivée à Québec de Louis Hébert, reconnu comme étant le premier apothicaire canadien. Quelques siècles s'écoulèrent ensuite avant que n'apparaisse une spécialité : la pharmacie hospitalière. Or, aujourd'hui, près de $17 \%$ de tous les pharmaciens au Canada s'efforcent d'assurer et d'améliorer la gestion des médicaments et les soins pharmaceutiques des patients en milieux hospitaliers. La Société canadienne des pharmaciens d'hôpitaux $(\mathrm{SCPH})$ soutient le progrès de la pratique en pharmacie hospitalière et l'atteinte des normes les plus élevées depuis près de 75 ans (jalons de la SCPH : https://www.cshp.ca/our-history ).

Aujourd'hui, le personnel et les bénévoles de la $\mathrm{SCPH}$ travaillent avec ardeur pour faire en sorte que les pharmaciens d'établissements soient en mesure d'exercer pleinement leur champ de pratique en contexte de collaboration et de produire un système de santé plus fort, le tout dans le but ultime d'optimiser la santé des patients. Dans la dernière année, nous avons formulé des demandes de consultation auprès de Santé Canada et d'autres groupes de professionnels en pharmacie et en médecine sur des sujets d'importance pour notre profession et nous avons aussi répondu à de telles demandes. À la fin 2016, par exemple, j'ai eu la chance d'offrir l'expertise et le point de vue des pharmaciens d'hôpitaux au Groupe consultatif de citoyens sur l'assurance-médicaments publique au Canada. Cette initiative avait pour but de contribuer de façon constructive au débat sur l'avenir du financement public des médicaments d'ordonnance. Les recommandations du groupe consultatif quant à la mise en œuvre d'un régime national d'assurance médicaments ont été communiquées à la ministre fédérale de la Santé et à ses homologues des provinces (https://open.library.ubc.ca/cIRcle/ collections/facultyresearchandpublications/52383/items/1.0340088). Par la suite, la SCPH a consulté ses membres et mis à jour sa déclaration de principes sur le sujet.

Après environ sept ans de travail avec le Bureau des substances contrôlées, la SCPH était heureuse d'apprendre en mars 2017 que, en vertu de l'article 56 de la Loi réglementant certaines drogues et autres substances, Santé Canada a accordé une exemption aux praticiens qui prescrivent de la méthadone à des patients hospitalisés. Ainsi, les praticiens n'ont plus à obtenir une exemption temporaire pour continuer à traiter les patients hospitalisés qui prenaient de la méthadone avant leur admission. Pour obtenir de plus amples renseignements, rendez-vous sur le site Web de Santé Canada (http://www.hc-sc.gc.ca/hc-ps/ substancontrol/pol/pol-docs/methadone-exemption-methadonefra.php).

Plus récemment, la SCPH participait à plusieurs initiatives liées à la crise des opioïdes. En collaboration avec la ministre fédérale de la Santé, la SCPH s'est engagée à concourir à la Déclaration conjointe sur les mesures visant à remédier à la crise des opioïdes (https://www.canada.ca/fr/sante-canada/services/ toxicomanie/conference-opioides/declaration-conjointe-mesuresvisant-remedier-crise-opioides.html). Dans la foulée de cet objectif, le 12 juin 2017 à Vancouver, nous avons participé, en compagnie de la ministre fédérale de la Santé, à une table ronde sur la crise des opioïdes organisée par SoinsSantéCAN lors de la Conférence nationale sur le leadership en santé. Les participants ont fait part de leurs connaissances et de leurs expériences des meilleures pratiques au sujet de la prévention, de la réduction des méfaits ainsi que de la gestion de la toxicomanie.

Alors que mon mandat de trois ans comme délégué présidentiel à la SCPH tire tranquillement à sa fin, je me surprends à réfléchir à ma propre expérience et aux réalisations de la SCPH pendant cette période. Je suis reconnaissant d'avoir eu la chance d'appartenir à la direction de cet organisme et d'avoir pu travailler avec autant de leaders exceptionnels en pharmacie hospitalière. C'est un honneur pour moi et je demeure tout aussi heureux de faire partie de cette merveilleuse profession. Nous avons fait tellement de chemin au cours des dernières années, mais notre route se poursuit et il y a toujours d'autres défis à relever. Notre avenir semble radieux et la SCPH continuera très certainement de jouer un rôle important dans le progrès continuel de la pharmacie hospitalière. Faites en sorte de participer et de soutenir personnellement la SCPH dans ce travail.

Etre pleinement présent est la meilleure garantie d'un avenir radieux. -Guy Finley

[Traduction par l'éditeur]

Glen J Pearson, B. Sc., B. Sc. Phm., Pharm. D., FCSHP, FCCS, est président sortant et agent de liaison externe pour la Société canadienne des pharmaciens d'hôpitaux. 